\title{
Hyperinsulinemia and hyperleptinemia , a possible threat for metabolic cardiovascular syndrome in obese prepubertal children.
}

\author{
Hedef Dhafir El-Yssin \\ (Professor, PhD. Post Doctorate/ member of the society for endocrinology, Bristol, UK \\ Ghazwan Talal Mehdi (MSc.) \\ *Department of Biochemistry, College of Medicine, University of Baghdad, Baghdad, Iraq
}

\begin{abstract}
Background: Childhood obesity is a condition where excess body fat negatively affects child's health or wellbeing. Due to the rising prevalence of obesity with its many adverse health effects in children, it has been recognized as a serious public health concern. Adipose tissue or body fat is composed of adipocytes specialized in storing energy as fat. Adipose tissue also serves as an important endocrine organ by producing hormones such as leptin, resistin, and the cytokine.
\end{abstract}

Aim: to explore the relationship between serum insulin and leptin in order to detect the presence of any impairment of these parameters as risk factors for metabolic cardiovascular syndrome in obese prepubertal children, in an attempt to overcome the increased health risk associated with obesity in children.

Subjects and methods: Fifty healthy obese children and 30 normal weight children who were selected from subjects attended the hospital and students of primary schools age range of children was between 5 to 11 years. Sera were obtained after centrifugation at $4000 \mathrm{rpm}$ for 15 minutes and were used to measure the levels of insulin and leptin.

Results: Insulin was significantly higher in obese children than in leans. However leptin was increased in obese but with no significance when compared to leans.

Conclusion: Obesity in children increases the risk of metabolic syndrome with hyperinsulinemia and hyper leptinemia. However with proper intervention hyperinsuilinemia could be reversible.

In the light of these results one should take intervention measures which are necessary to prevent excessive increase in weight during childhood.

\section{Introduction}

Childhood obesity is a condition where excess body fat negatively affects child's health or wellbeing. Due to the rising prevalence of obesity with its many adverse health effects in children, it has been recognized as a serious public health concern. Adipose tissue or body fat is composed of adipocytes specialized in storing energy as fat (1). Adipose tissue also serves as an important endocrine organ by producing hormones such as leptin, resistin, and the cytokines. (2)

Leptin is a peptide hormone encoded by the obesity gene. It is a $16-\mathrm{kDa}$ protein produced primarily in adipose tissues. It plays an important role in regulating food intake, reproduction, and immune function. (3). First described in 1994, leptin has proven to be a key metabolic protein with actions throughout the body (4) . Analogous to insulin, plasma leptin levels are directly correlated with adiposity. Circulating leptin is transported into the brain, where leptin receptors exist in many areas (5).

Insulin is a very important hormone for regulating the level of glucose in the blood. This hormone is formed in the beta cells the pancreas. In response to the levels of glucose in the blood, these cells become activated to produce insulin. Insulin activation also facilitates the conversion of glucose to glycogen and fat.

In obesity the initial deposition of triglycerides occurs in the subcutaneous adipose tissue. As this increases in size insulin resistance will rise and limits further subcutaneous lipid accumulation. It has also been shown that metabolic syndrome suddenly increases in prevalence at high levels of insulin resistance and suggested that this is due to the diversion of lipids from the subcutaneous to the visceral depot (6).

Insulin resistance is typically defined as decreased sensitivity and/or responsiveness to metabolic actions of insulin that promote glucose disposal. This important feature of metabolic syndrome (MS), diabetes, obesity, glucose intolerance, and dyslipidemia is also a prominent component of cardiovascular disorders, including hypertension, coronary artery disease, and atherosclerosis, which are characterized by endothelial dysfunction (7).

However; surrogate measures of insulin resistance IR, including fasting plasma insulin, the fasting glucose-to- insulin ratio (8), Homeostasis model assessment (HOMA) of IR (HOMA-IR), and Quantitative Insulin Sensitivity Check Index (QUICKI) (9), have been developed that can be applied to single measurement 
of fasting insulin and glucose. These surrogates have been shown to correlate with direct gold-standard measures and are useful in defining MS and in predicting the development of CVD and DMT2 .

While the main signal for leptin expressing is glucose metabolism, it is insulin that derives glucose into the cells and even though insulin, under physiological conditions in humans, does not have a direct effect on leptin expression (10).

\section{Subjects and methods:}

Fifty healthy obese children and 30 normal weight children who were selected from subjects attended the hospital and students of primary schools age range of children was between 5 to 11 years.

The study was done with the approval of the medical ethical committee in the ministry of health and ministry of education (Directorate General of education in Diyala). The parents agreement for withdrawing blood from their children was also granted.

Five $\mathrm{ml}$ of venous blood were aspirated from each subject. Sera were obtained after centrifugation at $4000 \mathrm{rpm}$ for 15 minutes and were used to measure the levels of insulin and leptin.

Principle of assessment of insulin and leptin was by quantitative sandwich enzyme immunoassay technique (ELISA).

An aliquot of the patient sample containing endogenous insulin is incubated in a coated well with enzyme conjugate, which is an anti-insulin antibody conjucated with biotin. After incubation the unbound conjugate is washed off. During the second incubation step streptavidin peroxidase enzyme complex binds to the biotin -anti-insulin antibody. The amount of bound HRP complex is usually proportional to the concentration of insulin in the sample. Having added the substrate solution ,the intensity of the color developed is measured which is proportional to the concentration of insulin in the patient sample. The kit was supplied by Demditec Diagnostics GmbH(Germany).

This technique include antibody specific for leptin pre-coated onto a microplate. Standards and samples are pipetted into the wells and any leptin present is bound by the immobilized antibody. After removing any unbound substances, a biotin-conjugated antibody specific for leptin is added to the wells. After washing, avidin conjugated Horseradish Peroxidase (HRP) is added to the wells. Following a further wash to remove any unbound avidin-enzyme reagent, a substrate solution is added to the wells and a color develops in proportion to the amount of leptin bound in the initial step. The color development is stopped by alterating the $\mathrm{pH}$, then intensity of the color is measured.

Anthropometrical Measurements: Body mass index (BMI) is a measure used to determine children's obesity. It is calculated using a child's weight and height. BMI does not measure body fat directly, but it is a reasonable indicator of body fatness for most children and teens.

A child's weight status is determined using an age- and sex-specific percentile for BMI rather than the BMI categories used for adults because children's body composition varies as they age and varies between boys and girls.

Centers for Disease Control and Prevention (CDC) Growth Charts are used to determine the corresponding BMI-for-age and sex percentile. For children and adolescents (aged 2-19 years):

A- Obesity is defined as a BMI at or above the 95th percentile for children of the same age and sex.

B- Normal is defined as a BMI at or above the 5 th to 85 th percentile for children of the same age and sex .
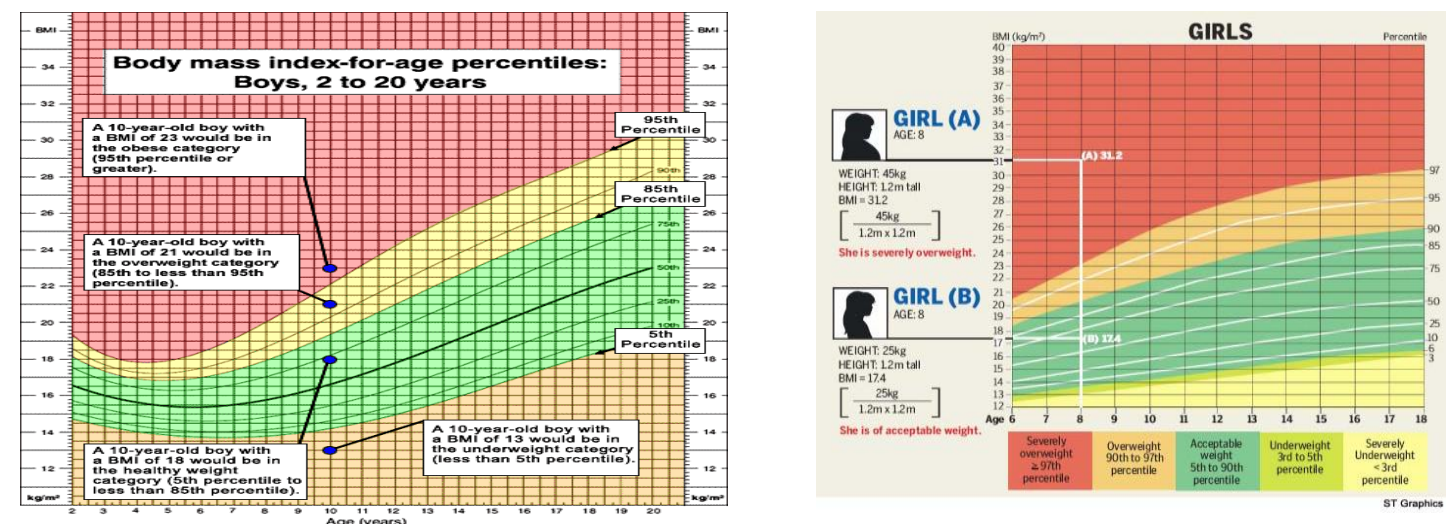

Figure (1): Centers for Disease Control and Prevention (CDC) Growth Charts for male and female children (www.cdc.gov/growthcharts/) 
Data were entered and analyzed using SPSS version 16 (SPSS Inc, USA ) for windows ( Microsoft corporation, USA ). Continuous variable were expressed as mean \pm SD, while categorical variable were expressed as a percentage and $95 \%$ CI . T - test, Anova. The level of significance for all statistical test was set at 0.05 .

\section{Results and discussion}

Table 1 and figure (2) summarize the results of both obese and lean weight children.

Table 1: Mean , standard Error mean, sig (2-tailed) of the Parameters studied in the two groups

\begin{tabular}{|l|l|l|l|}
\hline & Obese & lean & \\
& $($ Mean \pm SEM $)$ & $($ Mean \pm SEM $)$ & $P$ \\
\hline Insulin & $29.29 \pm 2.73$ & $17.08 \pm 1.91$ & 0.002 \\
\hline Leptin & $14.2 \pm 0.42$ & $13.6 \pm 0.75$ & 0.463 \\
\hline
\end{tabular}

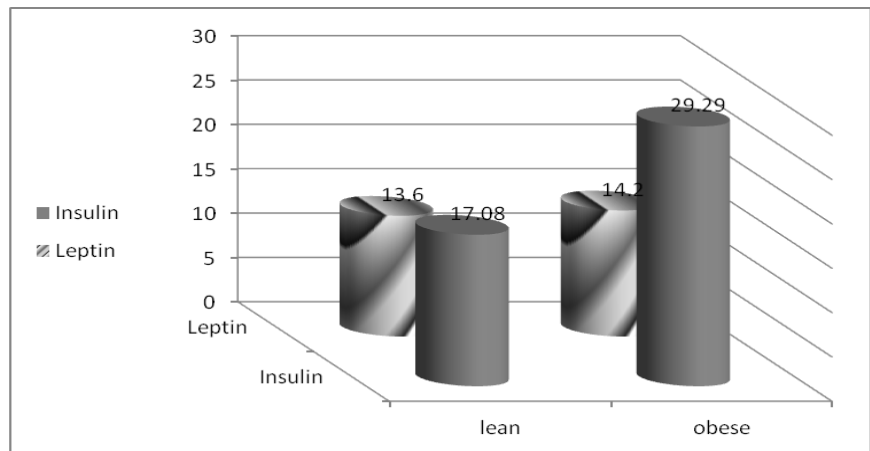

Figure(2): Mean values of the Parameters studied in the two groups

As shown in table (1) there is a significant difference between obese and normal weight children in the mean value of Insulin being high in obese (Mean 29.29) but low in normal weight children (Mean 17.08, $\mathrm{P}<0.002$ ). The results show that these children will predispose to insulin resistance and the development of DM.

Insulin resistance in obese children is strongly associated with specific adverse metabolic factors (11) .The one factor that should be monitored in obese children is their resistance to insulin, since it appears that the greater the level of insulin resistance, the greater the number of cardiovascular risk factors, the higher the prevalence of MS and, consequently, the greater the risk of premature development of DMT2 and CVD (12). Hyperinsulinemia is considered as an independent risk factor for CVD, since it plays an important role in the development of other components of MS, such as dyslipidemia, hypertension and hyperuricemia elevated levels of plasma insulin and an unfavorable lipids profile, in obese children, could be an indication that insulin resistance may be taking hold in some of the obese children, triggering off others of the factors that comprise a diagnosis of MS and exposing them to further risk factors. (13).

Insulin resistance in type 2 diabetics is associated with increased prevalence of coronary heart disease, blood coagulability, and dislipidemia leading to the suggestion that reducing insulin resistance in diabetics may reduce their tendency to develop thrombosis and hence coronary heart disease risk. In post- myocardial infarction patients, the metabolic syndrome and diabetes were prevalent and associated with increased risk of cardiovascular events and death (14).

On the other hand table (1) shows a non significant elevation of leptin serum levels in obese when compared to leans.

Leptin inhibits NPY/AgRP neurons and activates POMC/CART neurons, resulting in reduced food intake and increased energy expenditure (15). The effects of gut satiation signals such as CCK can be amplified by leptin which acts in the CNS, including the ARC in particular (16).

There are three types of leptin receptors identified: long, short, and secreted form. Among those, Ob$\mathrm{Rb}$ receptor, which is highly expressed in the hypothalamus, is thought to act as the main receptor involved in appetite control. Subcutaneous administration of recombinant leptin reduces fat mass. (3). However, obese individuals often have high leptin levels, which result in a failure to respond to exogenous leptin. This leptin resistance severely limits the therapeutic utility of leptin, and it is likely to result from reduced leptin receptor signal transduction (17). 
Circulating levels of insulin and leptin positively correlate with adipose tissue mass within the body. Both insulin and leptin are implicated in the long-term regulation of energy balance. Insulin is synthesized in the $\beta$ cells of the pancreas and is secreted rapidly after a meal, with well characterised hypoglycaemic effects. However, insulin also acts as an anorectic signal within the CNS. Insulin enters the CNS through a saturable and receptor-mediated transport process (18).

\section{Conclusion}

Obesity in children increases the risk of metabolic syndrome with hyperinsulinemia and hyper leptinemia. However with proper intervention hyperinsuilinemia could be reversible.

In the light of these results one should take intervention measures which are necessary to prevent excessive increase in weight during childhood.

\section{References}

[1] Sweeting HN. "Measurement definitions of obesity in childhood and adolescence: A field guide for the uninitiated “ . Nutr J 2007; 6:32.

[2] Cannon Band and Nedergaard J " Neither fat nor flesh." Nature. Developmental biology; 2008; 21; $947-948$.

[3] Farooqi IS and O’Rahilly S. Leptin: a pivotal regulator of human energy homeostasis. Am J Clin Nutr. Mar. 2009;89(3):980S984S.

[4] Zhang Y, Proeca R, Maffei M, Barone M, Leopold L, Friedman J. Positional cloning of the mouse obese gene and its human homologue . Nature 1994; 425-432.

[5] Banks WA, Kastin AJ, Huang Wea. Leptin enters the brain by a saturable system independent of insulin. Peptides 1996; 17:305311.

[6] Ali A.T., et al. Insulin resistance in the control of body fat distribution: A new hypothesis. Hormone me-tabolism Research 2011; 43:77-80.

[7] Kim J.; Montagnani M.; Koh K. et al.,. Reciprocal relationships between insulin resistance and endothelial dysfunction: molecular and pathophysiological mechanisms.Circulation 2006;113:I888-1904.

[8] Legro R.; Finegood D.; Dunaif A. A fasting glucose to insulin ratio is a useful measure of insulin sensitivity in women with polycystic ovary syndrome. J Clin Endocrinal Metab1998; 83: 2694-2698.

[9] Yokoyama H.; Emoto M.; Fujiwara S. Quantitative insulin sensitivity check index and the reciprocal index of homeostasis model assessment in normal range weight and moderately obese type 2 diabetic patients. Diabetes Care 2003; 26:2426-2432.

[10] Seufert J. Leptin effect on pancreatic beta-cell gene expression and function. Diabetes 2004; 53. (Suppl.1): S 152-8.

[11] Calle EE, Thun MJ, Petrelli JM, Rodriguez C, Heath CW Jr. Body-mass index and mortality in a prospective cohort of U.S. adults. N Engl J Med 1999;341:1097-105.

[12] Weiss R, Dziura J, Burgert TS, TamborlaneW, Taksali SE, Yeckcel CW, et al. Obesity and the metabolic syndrome in children and adolescents. N Engl J Med. 2004;350:2362-74.

[13] Sung RY, Tong PC, Yu CW, Lau PW, Mok GT, Yam MC, et al. High prevalence of insulin resistance and metabolic syndrome in overweight / obese preadolescent Hong Kong Chinese children aged 9 - 12 years. Diabetes Care. 2003;26:250-1.

[14] Levantesi G, Macchia A, Marfi si RM, et al. 2005. Metabolic syndrome and risk of cardiovascular events after myocardial infarction. J Am Coll Cardiol, 46:277-83.

[15] Sahu A., "Leptin signaling in the hypothalamus: emphasis on energy homeostasis and leptin resistance," Frontiers in Neuroendocrinology, 2003, vol. 24, no. 4, pp. 225-253.

[16] Blevins J. E. and Baskin D. G., "Hypothalamic-brainstem circuits controlling eating," Forum of Nutrition, 2010,vol. 63, pp. 133140.

[17] Munzberg H, "Leptin-signaling pathways and leptin resistance,” Forum of Nutrition, 2010, vol. 63, pp. 123-132.

[18] Baura G. D, Foster D M, Porte D. Jr. et al., "Saturable transport of insulin from plasma into the central nervous system of dogs in vivo. A mechanism 